\title{
A temperature compensation method of measuring frequency for cylindrical vibratory gyroscope in frequency split trimming
}

\author{
Youwang $\mathrm{Hu}^{1,2, a}$, Kai Zeng ${ }^{1,2}$, Haofang Wang ${ }^{1,2}$, Chang Liang ${ }^{1,2}$, Yongping Zhou ${ }^{1,2}$ and Xiaoyan Sun ${ }^{1,2}$ \\ ${ }^{1}$ The State Key Laboratory of High Performance and Complex Manufacturing, Central South University, Changsha, 410083, China \\ ${ }^{2}$ College of Mechanical and Electrical Engineering, Central South University, Changsha, 410083, China
}

\begin{abstract}
In the process of frequency split trimming, the fluctuation of environmental temperature causes a deviation in measuring the natural frequency, which is bigger than the request trimming accuracy. Therefore, a measuring method with temperature compensation is proposed to reduce this impact. Firstly, the relationship between natural frequency of resonator and environmental temperature are studied by experiments. Furthermore, the corresponding model of temperature compensation is proposed, which is written into the measuring unit to calculate the compensated frequency. The frequencies before and after temperature compensation are measured to prove the correctness of this method. At last, a real time measuring experiment is accomplished to verify the feasibility in frequency split trimming. Results show that the effect of temperature on the natural frequency reduced significantly with the temperature compensation measuring method. The proposed method is applicable for other types of vibratory gyroscope in precision frequency measurement.
\end{abstract}

\section{Introduction}

Cylindrical vibratory gyroscope is a kind of Coriolis Vibratory Gyroscope, which works with the Coriolis Effect of the elastic wave in the cylindrical resonator [1]. This kind of gyroscope has many advantages such as long service life, high operation accuracy, high reliability, small size, strong shock resistance, and low cost [2]. Therefore, these advantages render the cylindrical gyroscope useful in numerous applications such as avionics systems, borehole surveying, missiles, naval equipment, platform stabilization, and robotics [3]. However, low frequency split is of great importance for the performance of gyroscope, and it is the main quadrature error that should be reduced [4].

During the fabrication of gyroscopes, there are some unavoidable processing defects such as mass imbalances and geometric errors which may causes frequency split between the resonator's two working vibration modes [5]. Several approaches to decrease frequency split have been proposed by modelling and simulating on different kinds of gyroscopes [6-8]. Yi Tao et al presented a precision trimming method based on cup-button trimming for cylindrical vibratory gyroscope [9], and Kai Zeng et al proposed a resonator-top trimming method [10]. In these trimming methods, frequency measurement plays an important role to conduct precision trimming.

A frequency response analyzer was used to measure the frequency of MEMS vibratory gyroscopes [11], which is also available for cylindrical vibratory gyroscope. Yongmeng Zhang et al presented a method to improve the vibration stability of vibrating axis under temperature variation [12], however the effect on natural frequency of temperature fluctuation is ignored. An acoustic method for measuring the frequency of vibratory shell gyroscope was proposed by Xiang Xi et al, but they also referred that this method may be affected by the environmental noises such as the temperature fluctuation [13]. As is referred in references, frequency of gyroscope is very sensitive to temperature [14-16]. However, researches on sensitivity is rare, and a measurement method with temperature compensation is necessary for measuring.

A frequency measuring method with temperature compensation is proposed to reduce the influence of temperature fluctuation in this paper. It has been found that the relationship between frequency and temperature is linear by experiments, and a kind of linear compensatory measuring model is presented. The sensitivity for driving mode and sensing mode are $0.050 \mathrm{~Hz} /{ }^{\circ} \mathrm{C}$ and $0.051 \mathrm{~Hz} /{ }^{\circ} \mathrm{C}$ respectively. Based on this model, the deviation of measured frequency, which is caused by temperature fluctuation, can be reduced distinctly. After compensation, the experimental results show that the frequency is almost unchanged with the increase of temperature when the temperature changes slowly.

\section{Structure and measuring model}

\subsection{Structure}

Figure 1 shows the configuration of cylindrical vibratory gyroscope. It mainly consists of a cylindrical resonator

a Corresponding author: huyw@csu.edu.cn 
with eight piezoelectric electrodes equipped on its bottom and a circuit board that shows detail in figure 2. The vibration of resonator is excited and then converted to a voltage signal via the piezoelectric electrodes.
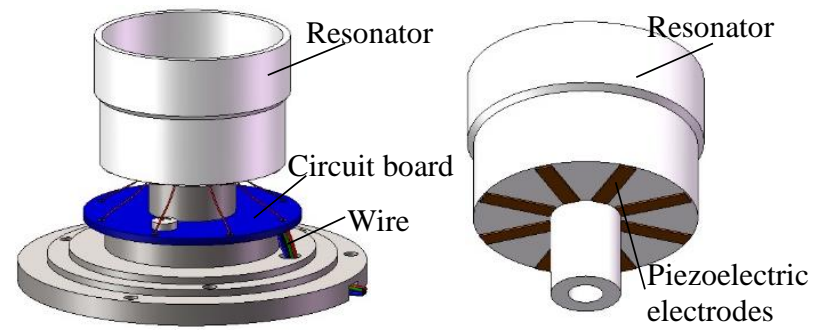

Figure 1. The configuration of cylindrical vibratory gyroscope

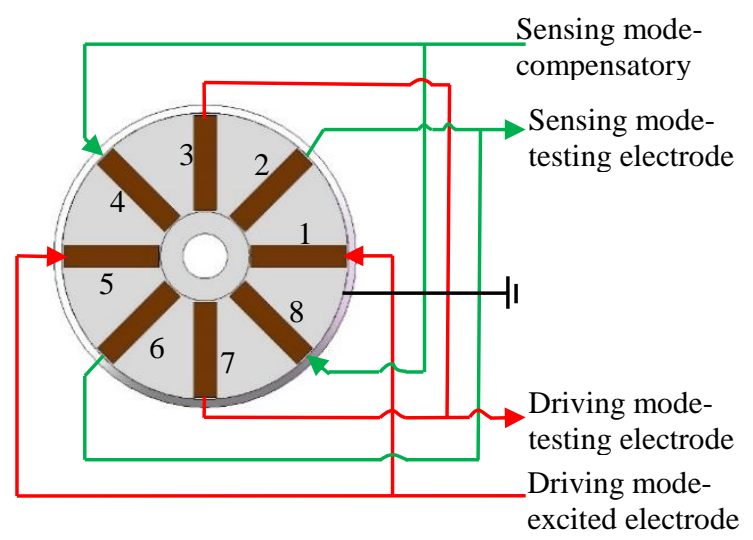

Figure 2. Principle of circuit board

Principle of the circuit board is presented in figure 2, there are 8 electrodes (4 pairs) glued on the bottom of resonator. The excited electrodes make resonator vibrated, and testing electrodes of sensing mode and driving mode are used for measuring frequency and amplitude of voltage. When amplitude reach the maximum, the corresponding frequency of input voltage is regarded as natural frequency. The last pair is compensatory electrode, which can compensate the result of testing electrode for sensing mode.

Natural frequency of sensing mode and driving mode cannot be measured simultaneously due to the structure of circuit board. In order to measure natural frequency, corresponding mode should be driven to vibrate. According to the working principle of cylindrical vibratory gyroscope, the sensing mode would not vibrate when there is not angular velocity. That is to say, after inputting alternating current in excited electrode of driving mode, only driving mode's natural frequency can be detected.

A method to measure sensing mode's natural frequency in trimming process is take compensatory electrode as excited electrode to drive sensing mode, and natural frequency of sensing mode can be detected in sensing mode's testing electrode. Furthermore, when changing the detecting mode, the environmental temperature may also have an unexpected change because of the temperature fluctuation of atmosphere. Therefore, measuring environment of the two modes may be different, which would result in the measuring variation of the frequency split. In this case, it is necessary to minimize the influence on natural frequency caused by temperature.

\subsection{Measuring model}

D. Keymeulen et al researched the effect of temperature on MEMS vibratory rate gyroscope [17]. They came up with a linear relationship between the frequency and temperature of $0.093 \mathrm{~Hz} /{ }^{\circ} \mathrm{C}$. Therefore, it is assumed that there is also a linear relationship between the cylindrical vibratory gyroscope's natural frequency and environmental temperature.

Then a formula as $f=K t+B$ can be proposed to express the relationship between natural frequency $f$ and environmental temperature $t$, and $K$ and $B$ are corresponding coefficients that can be obtained by experiments. Knowing the relationship between frequency and temperature, a measurement method with temperature compensation is presented.

First of all, an environmental temperature $t_{0}$ should be determined as a benchmark, and the natural frequency in this temperature is $f_{0}=K t_{0}+B$. Secondly, calculating the compensated frequency $\Delta f$ for $f=K t+B$, and $f$ is the natural frequency when the temperature is $t$. According to the formula $\Delta f=f-f_{0}=K\left(t-t_{0}\right)$, the compensated natural frequency can be obtained by $f_{0}=f-K\left(t-t_{0}\right)$ when the temperature is $t$. Finally, programming this compensation to measuring unit to display the $f_{0}$ constantly. The $f_{0}$ is compensated frequency when the temperature is $t$, and it would not change with the increase of temperature theoretically.

\section{Experimental setup and design}

\subsection{Experimental setup}

As shown in figure 3, the experimental setup mainly consists of a cylindrical vibratory gyroscope, a driving \& sensing circuit, a temperature chamber, a power supply, and a computer with frequency measuring program.

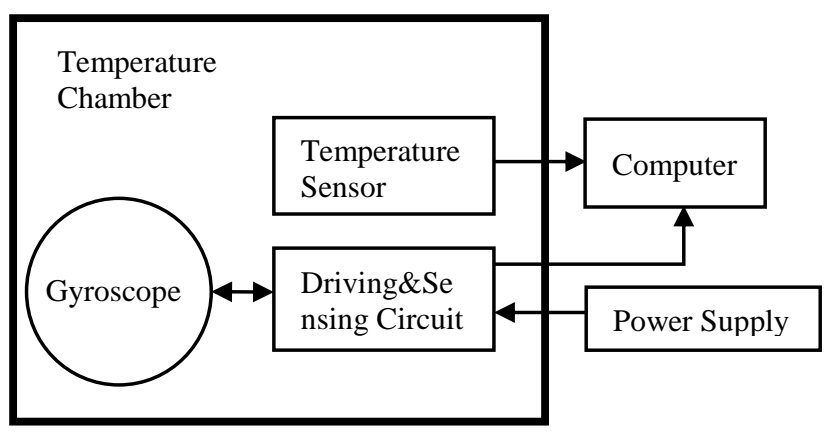



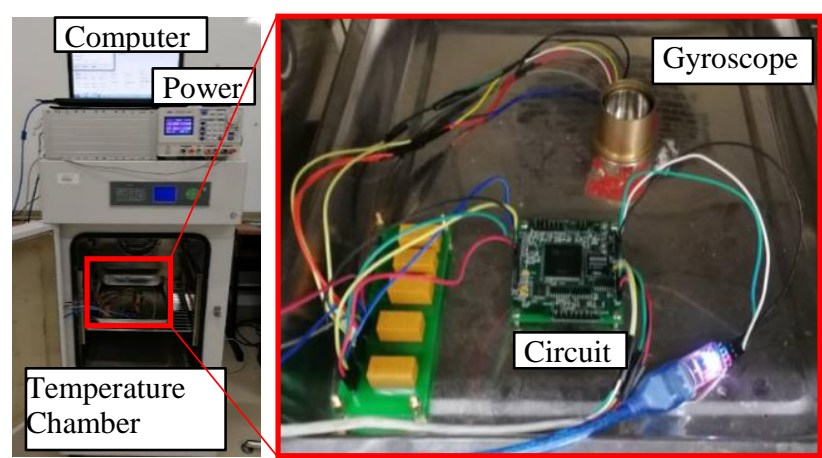

Figure 3. The schematic diagram and experimental system

The cylindrical vibratory gyroscope is placed on a horizontal stage with a cylindrical brass shell shielding the gyroscope to avoid the unexpected influence of unequal pressures from atmosphere. The frequency split trimming procedure is processing in a clean room where the temperature is around $20{ }^{\circ} \mathrm{C}$, and there still exists temperature fluctuation. To research the relationship between temperature and natural frequency, the temperature chamber $L H S-80 H C-I$ is chosen to change the temperature. A driving \& sensing circuit connected with eight piezoelectric electrodes is used for driving and sensing the vibratory modes, and a measuring program with the precision of $0.001 \mathrm{~Hz}$ to monitor the frequency is installed on the computer. The measurement of temperature is accomplished by temperature sensor $B S S$ $802 U$ which is connected to the same computer.

\subsection{Experimental design}

In order to obtain the frequency split of resonator, both the natural frequencies of driving and sensing modes need to be measured. However, as mentioned in Structure, there may be an unexpected fluctuation of the environmental temperature while changing the measuring mode. For each mode, experiments were settled to find out the frequency-temperature relationship. These experiments would be processed at the temperature chamber, a temperature range from $10^{\circ} \mathrm{C}$ to $30^{\circ} \mathrm{C}$ was set.

For each mode, the $10-30{ }^{\circ} \mathrm{C}$ range was separated averagely by 21 temperature points and the frequency of each temperature point was detected. There is one hour duration to keep the temperature uniform in chamber for each temperature point. At each point, the average of sampling temperature and frequency were taken to represent the point's temperature and frequency separately.

\section{Experimental results and discussion}

\subsection{Relationship between frequency and temperature}

The result of the frequency-temperature relationship is shown in figure 4 . Figure $4 \mathrm{a}$ shows that, for driving mode, the linearity is 0.998 and the slope is $0.050 \mathrm{~Hz} /{ }^{\circ} \mathrm{C}$. As show in figure $4 \mathrm{~b}$, the linearity is 0.999 and the slope is
$0.051 \mathrm{~Hz} /{ }^{\circ} \mathrm{C}$ for sensing mode. That is to say, the relationships between frequency and environmental temperature of both driving and sensing modes are linear. Besides, the slope of two modes are nearly equivalent, which means that the environmental temperature affect the frequency of both the two modes similarly.
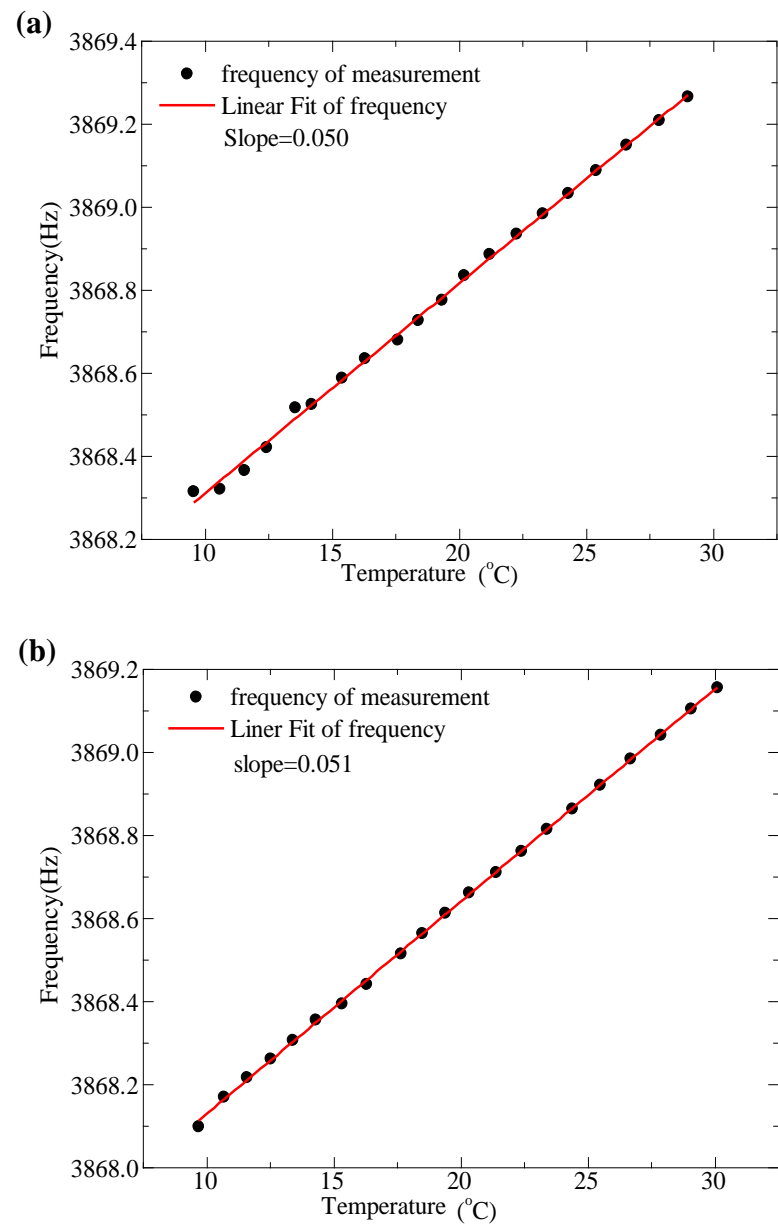

Figure 4. Relationship between frequency and temperature of driving (a) and sensing (b) modes

\subsection{Comparison of frequency before and after compensation}

For the formula $f_{0}=f-K\left(t-t_{0}\right)$, the fitted linear slope is the $K$, and $t_{0}$ is designed temperature. In the compensation experiment, the equipment requires a temperature of $20^{\circ} \mathrm{C}$, thus $t_{0}$ is set as $20^{\circ} \mathrm{C}$. When the measuring mode is driving mode, the $K$ is 0.050 , and $K$ is 0.051 while measuring mode is sensing mode. $t$ is the temperature detected by the sensor in real time. $f$ is the frequency detected by the measurement system without temperature compensation, and it is changed along with the increase of temperature. The compensated frequency $f_{0}$ is obtained from formula $f_{0}=f-K\left(t-t_{0}\right)$.

Then the formula can be converted into $f_{0 d}=f_{d^{-}}$ $0.050\left(t_{d}-20\right)$ and $f_{0 s}=f_{s^{-}}-0.051\left(t_{s}-20\right)$ for driving and sensing mode separately. The two formulas are written into the frequency measuring program, therefore the frequency before and after compensation can be obtained. In the temperature range of $10-30^{\circ} \mathrm{C}$, experiments are 
processed to measure $f_{0}$ and $f$, and the results are shown in figure 5 .
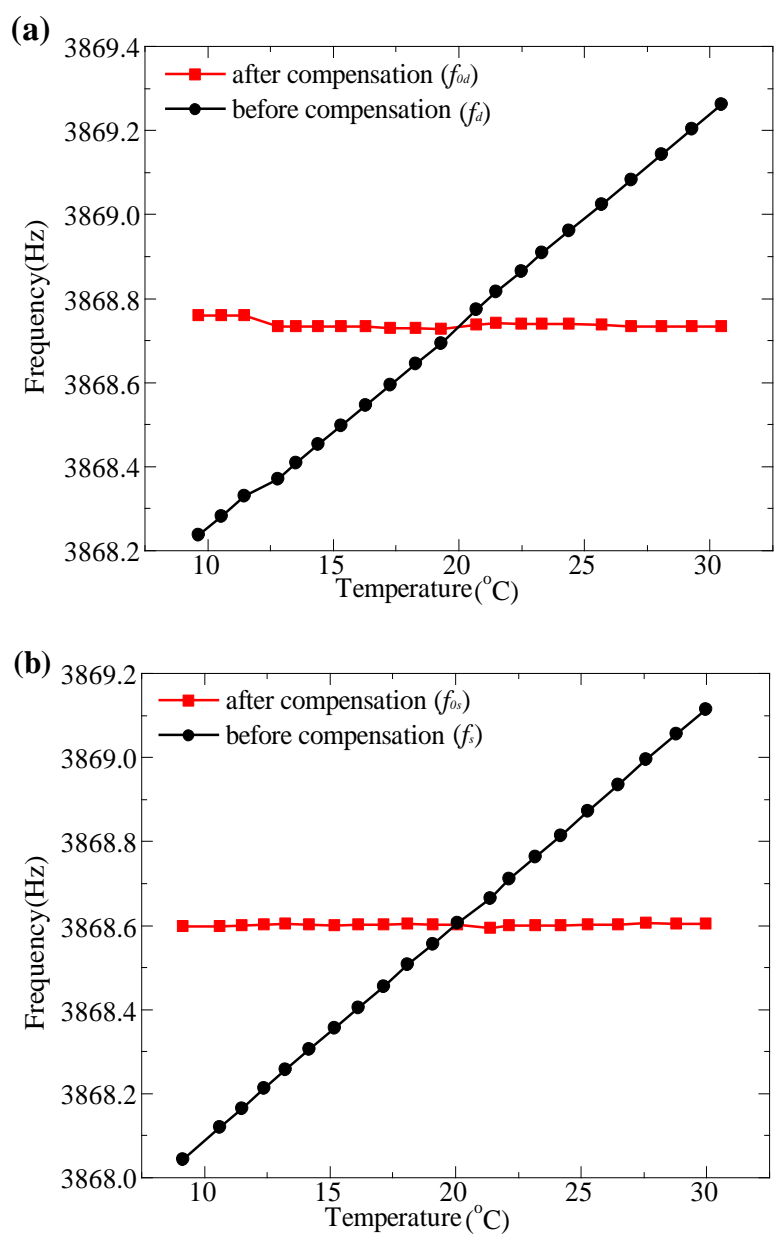

Figure 5. Frequency before and after compensation for driving (a) and sensing (b) modes.

As shown in figure 5 , the frequency $\left(f_{0}\right)$ after compensation is almost a straight line parallel to the horizontal axis, while the frequency $(f)$ before compensation is increasing along with the rise of temperature. When temperature is less than $20^{\circ} \mathrm{C}$, the measuring system added the compensation to $f$, and it reduced while temperature is higher than $20^{\circ} \mathrm{C}$. Under these circumstances, the natural frequency $f_{0}$ in $20^{\circ} \mathrm{C}$ is obtained regardless of temperature. That is to say, this measuring method nearly eliminate the effect of temperature on frequency.

\subsection{Experiments in real time}

Another experiment was completed by the same experimental equipment to test the stability of this measuring method in real time. The sensing mode was selected to be the experimental measuring mode, and the temperature of chamber was set to continuously fluctuate around $20^{\circ} \mathrm{C}$. The changing temperature is shown in figure $6 \mathrm{a}$, and figure $6 \mathrm{~b}$ presents the frequencies before and after compensation in real time.
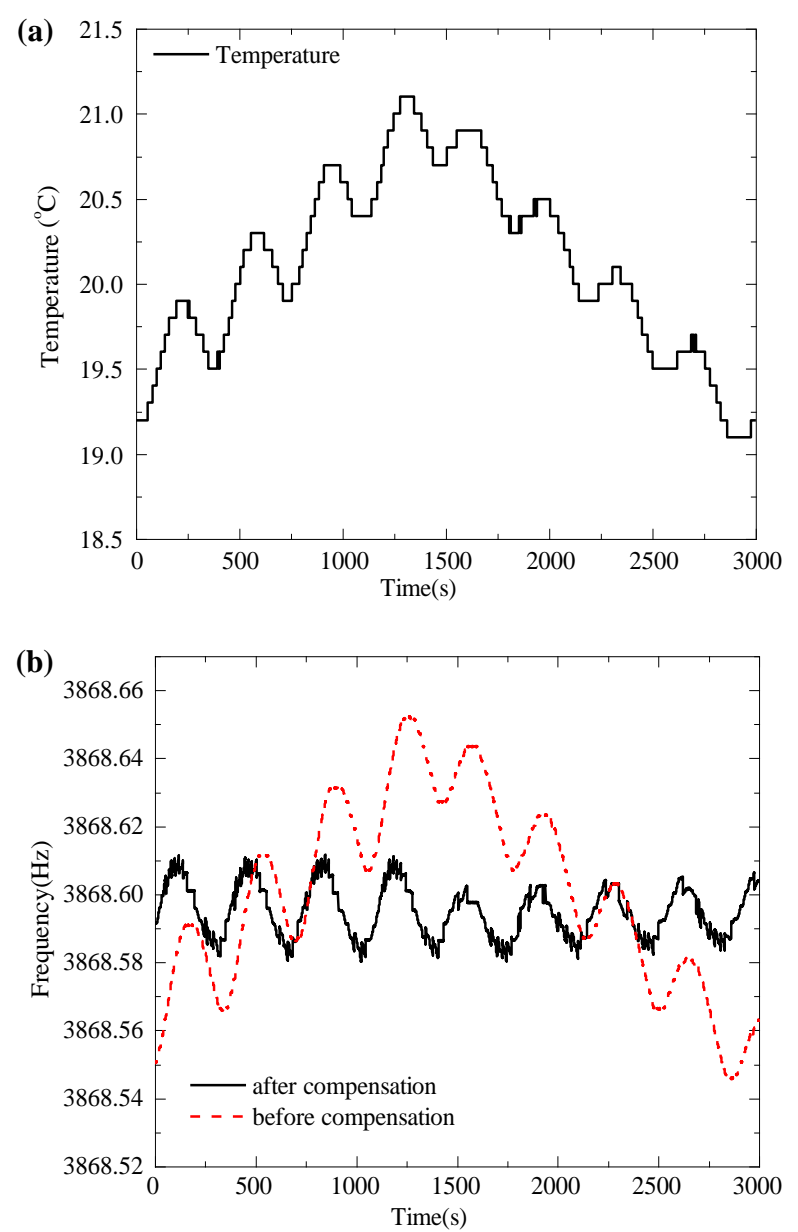

Figure 6. (a) Temperature fluctuation around $20^{\circ} \mathrm{C}$, (b) comparison of frequency before and after temperature compensation.

As shown in figure $6 \mathrm{a}$, the temperature in chamber increase firstly and then decrease on the whole. Besides, there is a small fluctuation in the process of increase and decrease. It is noteworthy that the temperature changes step by step, which owing to the sensitivity of the temperature sensor is $0.1{ }^{\circ} \mathrm{C}$. Under this condition, the measured frequency before and after compensation is show in figure $6 \mathrm{~b}$.

The frequency of sensing mode before and after compensation is showed in figure $6 \mathrm{~b}$, and it is obvious that the fluctuation of compensated frequency is much smaller than uncompensated frequency. The uncompensated frequency changes follow with the altering of temperature, while compensated frequency just fluctuates in a small scale. In addition, another phenomenon in figure $6 \mathrm{~b}$ is both the compensated and uncompensated frequency have a small fluctuation just as the small fluctuation of temperature in figure $6 \mathrm{a}$. The reason is that there is a lag in temperature between gyroscope and temperature sensor when the temperature changes quickly. The difference of temperature result in an offset in compensation, therefore the frequency still fluctuates. It is noteworthy that the fluctuation can be eliminated when the temperature of gyroscope is uniform and be measured without lag, such as the results of figure 5 . 


\section{Conclusion}

In summary, this paper presented a temperature compensation method to measure frequency for cylindrical vibratory gyroscope, and it can decrease the effect of temperature on frequency effectively. With this method, the accurate natural frequency can be obtained to conduct frequency split precision trimming.

More specifically, the measuring model with temperature compensation is proposed, and the effects of temperature on frequency is studied. In other words, the coefficients of temperature compensation for both driving and sensing modes are obtained respectively. Furthermore, the measured frequencies before and after compensation at each temperature point in the range of $10-30^{\circ} \mathrm{C}$ was compared to verify the correctness of the temperature compensation model and coefficient. In addition, in order to test the stability of this compensation method in real time, comparison before and after compensation was presented when the temperature was continuously changing around $20^{\circ} \mathrm{C}$.

In brief, these studies show that the relationship between natural frequency and temperature is linear, and the proposed model is suitable for cylindrical vibratory gyroscope. The measuring system with temperature compensation can decrease the effect of temperature on frequency significantly. It is worth mentioning that this system is also suitable for other kinds of vibratory gyroscope. It is believed that the compensated method will play an important role in frequency split trimming to improve the performance of gyroscope.

\section{Acknowledgements}

The authors would like to thank to the State Key Laboratory of High Performance and Complex Manufacturing, College of Mechanical and Electrical Engineering, Central South University, China, for technical support and access to equipment. The authors also would like to thank to the Laboratory of Microsystems, National University of Defense Technology, China, for technical support and access to equipment. This work was supported by the National Nature Science Foundation of China (Grant Nos. 51475481, 51475482, 51335011, 91323301), and the Fundamental Research Funds for the Central Universities of Central South University (2017zzts419).

\section{References}

1. C. Langmaid, Sensor Rev. 16, 14 (1996)

2. V. V. Chikovani, I. M. Okon, A. S. Barabashov ,P. Tewksbury, Position, Location \& Navigation Symposium, IEEE/ION 238 (2007)

3. A. K. Singh, Defence Sci. J. 57, 95 (2007)

4. P. Bisegna ,G. Caruso, J. Sound Vib. 306, 691 (2007)

5. J. A. Malluck ,S. N. Melkote, J. Manuf. Sci. E.-T. ASME 126, 141 (2004)

6. A. K. Rourke, S. Mcwilliam ,C. H. J. Fox, J. Sound
Vib. 248, 695 (2001)

7. A. K. Rourke, S. Mcwilliam ,C. H. J. Fox, J. Sound Vib. 256, 319 (2002)

8. S. Mcwilliam, J. Ong ,C. H. J. Fox, J. Sound Vib. 279, 453 (2005)

9. T. Yi, X. Xiang, D. Xiao, Y. Tan, H. Cui ,X. Wu, Chin. J. Mech. Eng.-En. 25, 63 (2012)

10. K. Zeng, Y. Hu, G. Deng, X. Sun, W. Su, Y. Lu ,J. A. Duan, Sensors-Basel 17, (2017)

11. Z. Hou, D. Xiao, X. Wu, P. Dong, Z. Niu, Z. Zhou, X. Zhang, IEEE International Conference on Nano/micro Engineered and Molecular Systems 293 (2011)

12. Y. Zhang, Y. Wu, X. Xu, X. Xi ,X. Wu, Int. J. Precis. Eng. Man. 18, 1813 (2017)

13. X. Xi, Y. Wu, Y. Zhang, X. Wu, Y. Zheng, X. Wu, IEEE Sens. J. 14, 4069 (2014)

14. J. Fang ,J. Li, IEEE T. Instrum. Meas. 58, 2923 (2009)

15. Y. Wu, X. Xi, Y. Tao, X. Wu ,X. Wu, Sensors-Basel 11,7665 (2011)

16. V. Apostolyuk, V. Chikovani, Methods and Systems of Navigation and Motion Control 116 (2013)

17. D. Keymeulen, C. Peay, K. Yee ,D. L. Li, Aerospace Conference 1 (2005) 